\title{
Poly(furfuryl alcohol) nanospheres: a facile synthesis approach based on confinement effect of polymer and a template for synthesis of metal oxide hollow nanospheres
}

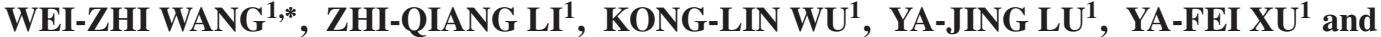 \\ XIN-JIE SONG ${ }^{2}$ \\ ${ }^{1}$ The Key Laboratory of Functional Molecular Solids, Ministry of Education, Anhui Laboratory of Molecular-Based \\ Materials, College of Chemistry and Materials Science, Anhui Normal University, Wuhu 241000, PR China \\ ${ }^{2}$ Department of Food Science and Technology, Yeungnam University, Gyeongsan 712749, Republic of Korea
}

MS received 7 July 2015; accepted 10 August 2015

\begin{abstract}
This paper describes a facile hydrothermal approach to the large-scale synthesis of well-dispersed poly(furfuryl alcohol) (PFA) nanospheres with an average diameter of $350 \mathrm{~nm}$ in the presence of poly(vinyl pyrrolidone) (PVP). Scanning electron microscopy and transmission electron microscopy studies showed that different morphologies of PFA could be obtained by adjusting the ratio of PVP and furfuryl alcohol (FA). As a whole, the results demonstrate that PVP plays a key role in controlling the polymerization process of FA. The confinement effect of PVP is proposed to explain the formation process of PFA nanospheres. Furthermore, the as-prepared PFA nanospheres have a functional surface that allow them to act as an ideal template for fabricating metal oxide hollow nanospheres.
\end{abstract}

Keywords. Poly(furfuryl alcohol); nanospheres; hydrothermal; hollow.

\section{Introduction}

Furfuryl alcohol (FA) is a product derived from crop residues made by reducing furfural. ${ }^{1}$ As a very reactive substance, it can form a black resin of poly(furfuryl alcohol) (PFA) through heated or acid-catalysed polymerization., ${ }^{2,3}$ FA is compatible with many organic polymers and inorganic materials, ${ }^{4}$ and it provides a high carbon yield when pyrolysed. Therefore, PFA is important for use in metalcasting molds, corrosion-resistant materials and negative photoresists. ${ }^{5}$ It has also been shown to act as a precursor to synthesize various carbon-based materials and polymer composites for a wide range of applications, such as adsorbents, separation membranes, electrodes of fuel cells, lithium batteries, electric double-layer capacitors, etc. ${ }^{6}$ Although there have been systematic studies on the polymerization mechanisms and condensation kinetics of PFA, ${ }^{7-9}$ little attention has been devoted to synthesize nanoscale PFA species, for example synthesis of PFA nanospheres. In recent years, the design and synthesis of polymer nanospheres has garnered the interest of researchers owing to their fundamental scientific importance and potential applications, ${ }^{10}$ including drug and DNA delivery and photonic band-gap crystal. ${ }^{11}$ Many approaches for preparing polymer nanospheres have been developed, which can be summarized into three

*Author for correspondence (wangwz@mail.ahnu.edu.cn) categories according to the preparation method: (i) as a conventional preparation method, emulsion or microemulsion polymerization can make polymeric particles in the size range of $10-10^{3} \mathrm{~nm} .{ }^{12,13}$ (ii) As a mimic of globular proteins, self-assembly of linear block copolymers has been used to prepare nanospheres with core-shell morphology. ${ }^{14,15}$ (iii) In recent times, a new method involving the collapse and intramolecular coupling of single-polymer chains to fabricate nanospheres has been proposed. ${ }^{16-18}$ Despite these advancements, it is still important and meaningful to explore convenient and facile methods to fabricate polymer nanospheres.

Hydrothermal process has been acknowledged as one of the most efficient methods for preparing inorganic nanomaterials with various morphologies. ${ }^{19}$ In this study, a facile polymer-assisted hydrothermal approach was employed to obtain well-dispersed PFA nanospheres. After investigating the effect factor on morphology of the products, a possible polymer confinement polymerization process was identified to explain the formation of PFA nanospheres. Since conventionally hydrothermal process can be used to prepare spherical PFA in nanoscale, it is believed that this is an effective approach because it is simple, low-cost and ideal for largescale production of polymer nanospheres. Furthermore, the surface of as-prepared PFA nanospheres is functionalized, which offer a beneficial environment for interactions with metal cations and subsequent conversion to metal oxide hollow nanospheres through removal of the PFA core. 


\section{Experimental}

\subsection{Preparation of PFA nanospheres}

All reagents were of analytical grade (Shanghai Chemical Reagents Co.) and used without further purification. A typical procedure was as follows: an aqueous solution of $40 \mathrm{ml}$ was first prepared by dissolving $1 \mathrm{~g}$ of poly(vinyl pyrrolidone $)(\mathrm{PVP})(\mathrm{K} 30$, polymerization degree $=360)$ in distilled water. Then, $0.5 \mathrm{ml}$ of FA was added dropwise into the former solution with constant stirring until a homogeneous clear solution was formed. The resulting solution was transferred into a Teflon-lined stainless-steel autoclave. The autoclave was sealed and maintained at $160^{\circ} \mathrm{C}$ for $12 \mathrm{~h}$. The reddish brown products were isolated by centrifugation (7000 $\mathrm{rpm}, 8 \mathrm{~min}$ ). Then, products were washed by cycles of dispersion/washing/centrifugation in distilled water and ethanol several times to remove excess PVP. Finally, the products were dried in a vacuum at $60^{\circ} \mathrm{C}$ for $6 \mathrm{~h}$.

\subsection{Preparation of $\alpha-\mathrm{Fe}_{2} \mathrm{O}_{3}$ hollow nanospheres}

A specific amount of as-prepared PFA nanospheres was well dispersed into $40 \mathrm{ml}$ of water with the assistance of sonication for $10 \mathrm{~min} . \mathrm{FeCl}_{3} \cdot 6 \mathrm{H}_{2} \mathrm{O}(1 \mathrm{mmol})$ was dissolved into the above solution with vigorous stirring for $30 \mathrm{~min}$; after which $3 \mathrm{ml}$ of $1 \mathrm{M} \mathrm{NaOH}$ aqueous solution was slowly added into the mixture with stirring. The mixture was kept at room temperature for $5 \mathrm{~h}$ with vigorous stirring. The products were collected by centrifugation and cleaned by three cycles of centrifugation/washing/redispersion in water and ethanol before being dried at $60^{\circ} \mathrm{C}$ for $4 \mathrm{~h}$. Finally, $\alpha-\mathrm{Fe}_{2} \mathrm{O}_{3}$ hollow nanospheres were obtained after calcination at $500^{\circ} \mathrm{C}$ in static air for $2 \mathrm{~h}$.

\subsection{Characterization}

Transmission electron microscopy (TEM) images of the asprepared products were taken on a Hitachi H-800 TEM at an accelerating voltage of $200 \mathrm{kV}$. Morphologies of the products were also characterized using a scanning electron microscope (SEM, JEOL-6300F). Fourier transform infrared (FT-IR) spectrum was recorded on a Bruker EQUINOX55 FT-IR spectrometer using wavenumbers of $500-4000 \mathrm{~cm}^{-1}$ at room temperature. The sample was pressed into disk with $\mathrm{KBr} .{ }^{1} \mathrm{H}$ NMR spectrum was measured with a Bruker AVANCE 300 spectrometer at a frequency of $300 \mathrm{MHz}$. The products were dissolved in $\mathrm{CDCl}_{3}$, and TMS was used as an internal reference.

\section{Results and discussion}

\subsection{Morphology and characterization of PFA nanospheres}

The morphology and size of the as-prepared products were examined by TEM and SEM. Figure $1 \mathrm{a}$ and $\mathrm{b}$ clearly
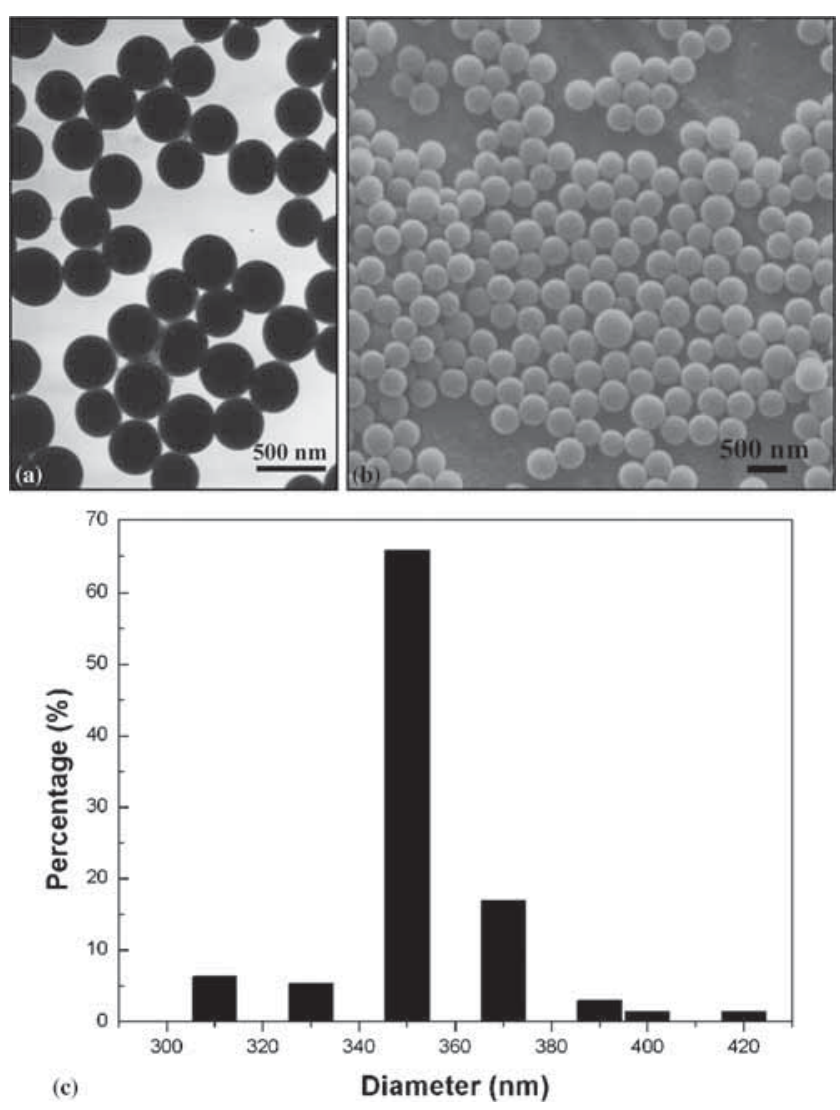

Figure 1. PFA nanospheres: (a) TEM and (b) SEM image. (c) Size distribution histogram of about 200 PFA nanospheres.

demonstrates that the products are composed of large quantities of nanospheres with very smooth surfaces and good dispersibility. Figure 1c shows a size distribution histogram of about 200 nanospheres from the SEM image, showing that these nanospheres have a narrow size distribution with an average diameter of $350 \mathrm{~nm}$. The dispersity of the asprepared nanospheres is reflected by the polydispersity index (PDI). The closer PDI is to 1 , the more monodisperse the particles are. ${ }^{20} \mathrm{PDI}$ is defined by the following: ${ }^{20-22}$

$$
\mathrm{PDI}=\frac{D_{\mathrm{w}}}{D_{\mathrm{n}}}, \quad D_{\mathrm{w}}=\frac{\sum n_{\mathrm{i}} D_{\mathrm{i}}^{4}}{\sum n_{\mathrm{i}} D_{\mathrm{i}}^{3}}, \quad D_{\mathrm{n}}=\frac{\sum n_{\mathrm{i}} D_{\mathrm{i}}}{\sum n_{\mathrm{i}}},
$$

$D_{\text {w }}$ is the weight average diameter, $D_{\mathrm{n}}$ the number average diameter and $n_{\mathrm{i}}$ the number of particles with diameter $D_{\mathrm{i}}$. The PDI of the as-prepared products is about 1.007, which indicates that these nanospheres have good dispersibility.

Figure 2a shows the FT-IR spectrum of the nanospheres, confirming they are PFA. The bands at 733, 1016, 1162, 1505 and $3122 \mathrm{~cm}^{-1}$ are attributed to stretching of the $=C-$ $\mathrm{O}-\mathrm{C}=$ and $=\mathrm{C}-\mathrm{H}$ groups of the furan rings. The bands at 788 and $1563 \mathrm{~cm}^{-1}$ are the result of bending out of the plane of the $\mathrm{CH}$ linkage and stretching of the $-\mathrm{C}=\mathrm{C}-$ groups in the $2-5$ disubstituted furan rings. The bands at 1357 and 1608 $\mathrm{cm}^{-1}$ are because of the ring stretch modes of the two substituted furan rings. The aliphatic $\mathrm{CH}_{2}$ groups in the polymer are responsible for the bands at 2920 and $1421 \mathrm{~cm}^{-1}$, 


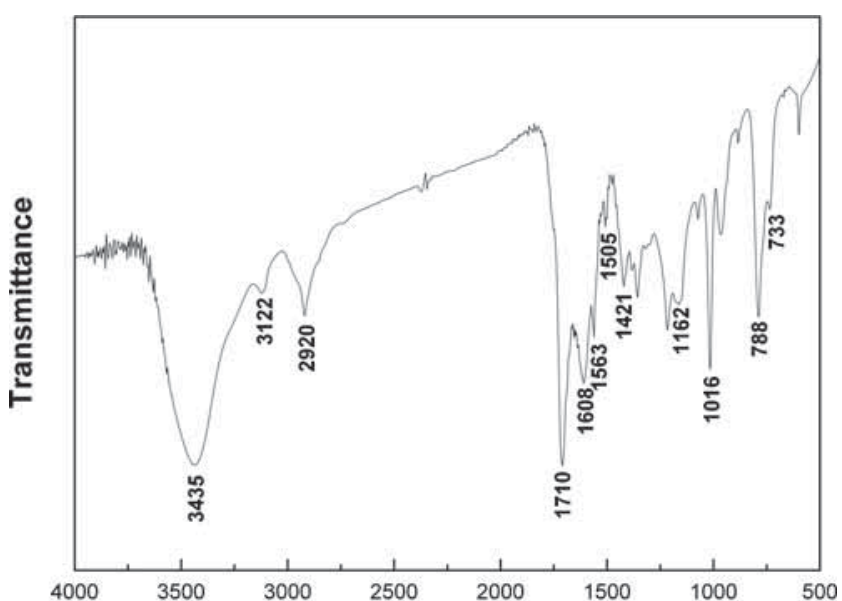

(a)

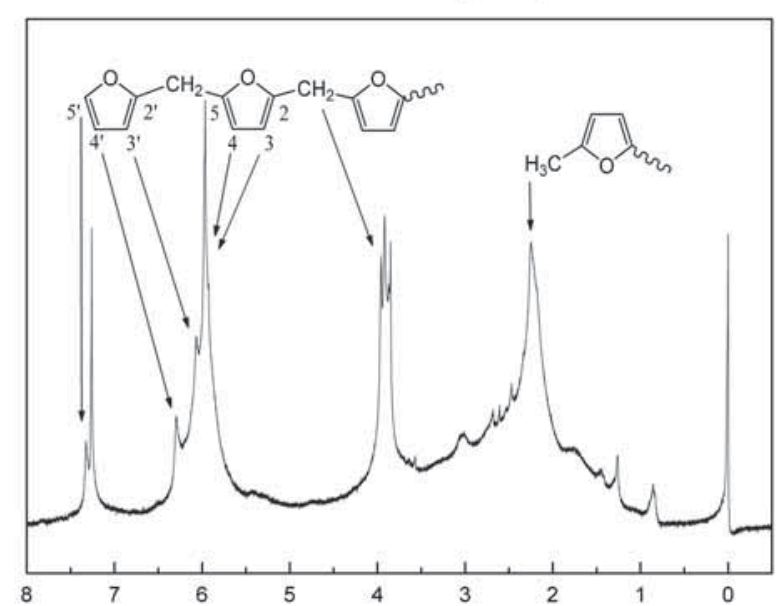

(b)

$\delta(\mathrm{ppm})$

Figure 2. (a) FT-IR spectrum and (b) ${ }^{1} \mathrm{H}$ NMR spectrum $(300$ $\mathrm{MHz}$, solvent: $\mathrm{CDCl}_{3}$ ) of PFA nanospheres.

whereas the band at $3435 \mathrm{~cm}^{-1}$ is assigned to stretching of the $\mathrm{OH}$ end groups in PFA. The absorption band at 1710 $\mathrm{cm}^{-1}$ is ascribed to carbonylic structures originating from opening of some furan rings by electrophilic attack. These characteristic absorption bands are similar to bulk PFA resin in the literature. ${ }^{23-25}$ There are no characteristic vibrational absorption signals for the $\mathrm{C}=\mathrm{O}$ bond of $\mathrm{PVP}$ at $\sim 1665$ $\mathrm{cm}^{-1}$ in the FT-IR spectrum, ${ }^{26}$ indicating that PVP was completely removed by repeated washing with distilled water and absolute ethanol.

The ${ }^{1} \mathrm{H}$ NMR spectrum of the PFA nanospheres was also checked in order to understand the polymerization of FA (see figure $2 \mathrm{~b}$ ). The proton signal at $7.33 \mathrm{ppm}$ corresponds to the terminal furan ring $\left(\mathrm{H}_{5^{\prime}}\right)$; signals between 5.80 and $6.40 \mathrm{ppm}$ can be assigned to the protons $\mathrm{H}_{3}$ and $\mathrm{H}_{4}$ of the furan ring, in addition to those arising from $\mathrm{H}_{3^{\prime}}(6.07 \mathrm{ppm})$ and $\mathrm{H}_{4^{\prime}}(6.30 \mathrm{ppm})$. The complex signals centred around $3.90 \mathrm{ppm}$ are due to the $-\mathrm{CH}_{2}-$ groups joined to the $\mathrm{C}_{2}$ and $\mathrm{C}_{5}$ positions of the two furan rings. The broad signals around $2.2 \mathrm{ppm}$ are assigned to $-\mathrm{CH}_{3}$ end groups attached
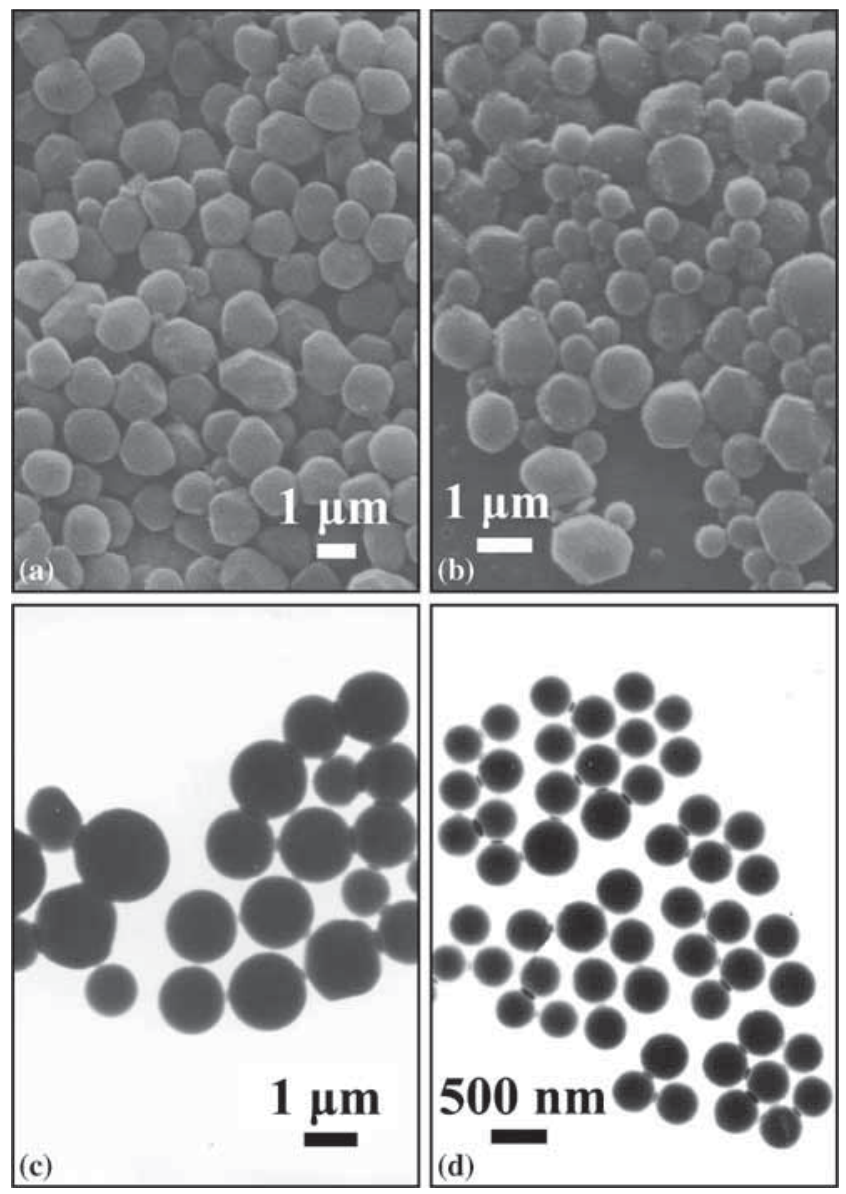

Figure 3. SEM and TEM images of PFA prepared with various ratios of PVP/FA $\left(\mathrm{g} \mathrm{ml}^{-1}\right)$ : (a) 0.4 , (b) 0.5 , (c) 1 and (d) 2.5 .

to the $C_{2}$ or $C_{5}$ position of the furan ring. ${ }^{27}$ This is not new since methylated FA oligomers have been isolated and characterized previously. ${ }^{28,29}$ The signals between 1.50 and 3.50 ppm indicate the presence of other aliphatic protons. ${ }^{30}$ The likely origin of the unassigned aliphatic signals is the opening of some furan rings. ${ }^{1}$ No signal is observed around 4.50 ppm, indicating the absence of detectable amounts of $-\mathrm{CH}_{2}-$ $\mathrm{O}-\mathrm{CH}_{2}-$ bridges in these polymers. ${ }^{24}$ Judging from the ${ }^{1} \mathrm{H}$ NMR spectrum, it can be concluded that the polymerization of FA mainly involves condensation of the $\mathrm{OH}$ group with the mobile hydrogen atom of the heterocycle at $\mathrm{C}_{5},{ }^{31}$ as indicated in equation (2).

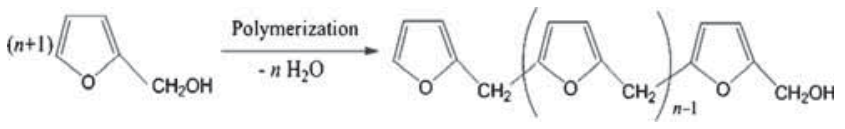

The above analyses demonstrate that the spherical PFA nanomaterials were successfully formed by polymerization of FA under a facile hydrothermal process. Since neither expensive reagents nor organic solvents are used in the synthesis of polymer nanospheres, this approach is cheaper, greener and easier in comparison with other techniques. 
Table 1. Morphology and size of PFA prepared using different ratios of PVP/FA.

\begin{tabular}{lcccc}
\hline PVP $(\mathrm{g})$ & FA $(\mathrm{ml})$ & Ratio $=$ PVP/FA $\left(\mathrm{g} \mathrm{ml}^{-1}\right)$ & Morphology & Size \\
\hline None & 0.5 & 0 & Bulk & - \\
0.1 & 0.5 & 0.2 & Bulk & - \\
0.2 & 0.5 & 0.4 & Polyhedron & $1.5-2 \mu \mathrm{m}$ \\
1 & 2 & 0.5 & Polyhedron + sphere & $0.5-1.5 \mu \mathrm{m}$ \\
0.5 & 0.5 & 1 & Sphere + polyhedron & $0.5-1.2 \mu \mathrm{m}$ \\
0.8 & 0.5 & 1.6 & Sphere & $\sim 400 \mathrm{~nm}$ \\
1 & 0.5 & 2 & Sphere & $\sim 350 \mathrm{~nm}$ \\
1 & 0.4 & 2.5 & Sphere & $\sim 350 \mathrm{~nm}$ \\
4 & 1 & 4 & Sphere & $\sim 400 \mathrm{~nm}$ \\
3 & 0.5 & 6 & Sphere & $\sim 400 \mathrm{~nm}$ \\
1 & 0.1 & 10 & Sphere & $\sim 280 \mathrm{~nm}$ \\
\hline
\end{tabular}
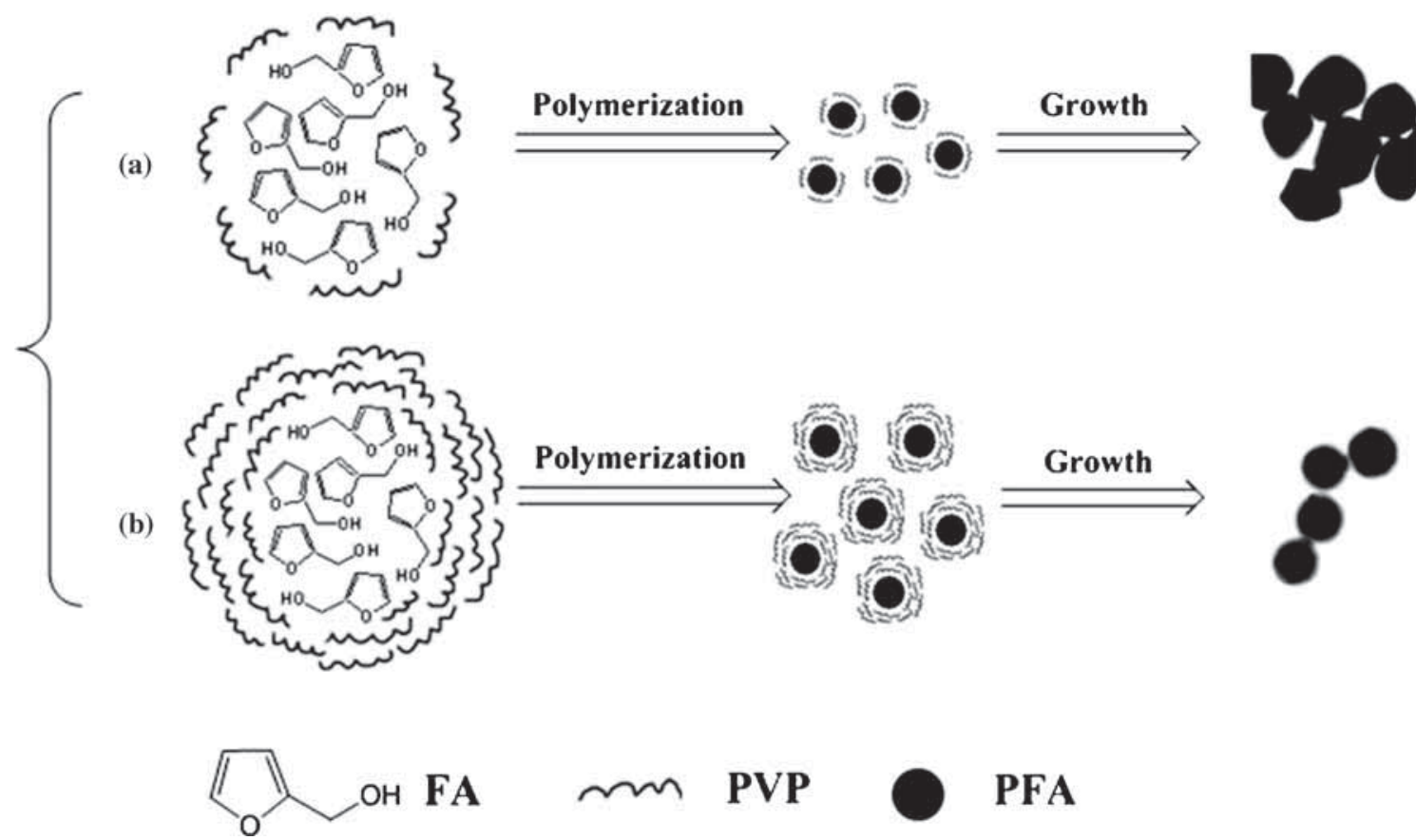

Figure 4. Schematic illustration of possible formation of PFA with different morphologies: (a) PVP is deficient and (b) PVP is excessive.

\subsection{Effect of ratio of PVP/FA}

In our experiment, PVP was shown to play a significant role in the formation of PFA nanospheres. When no PVP was added to our reaction system, no PFA nanospheres formed and only black bulk PFA resin was obtained. To understand the effect of PVP on polymerization of FA and the formation of PFA nanospheres, morphology and size of PFA were investigated using TEM and SEM under the same conditions except for different ratios of PVP/FA $\left(\mathrm{g} \mathrm{ml}^{-1}\right)$.

At a low PVP/FA ratio (0.2), main products were mainly bulk PFA resin. Increasing the amount of PVP to a PVP/FA ratio of 0.4 resulted in reddish brown precipitates. SEM image (figure 3a) reveals that products were large polyhedrons with sizes of about $1.5-2 \mu \mathrm{m}$. After increasing the PVP/FA ratio to 0.5, PFA nanospheres formed, but main products were still large polyhedrons (figure $3 b$ ). Further increasing the PVP/FA ratio to 1 also increased the proportion of PFA nanospheres (figure 3c). TEM image (figure 3d) shows that most of the products at a PVP/FA ratio of 2.5 were PFA nanospheres with an average diameter of $350 \mathrm{~nm}$, which is similar to typical products (ratio of 2). These images clearly demonstrate that the morphology of PFA could be developed from a large polyhedron to small sphere by increasing the ratio of PVP/FA. The detailed relationship between the PVP/FA ratio and morphology and size of PFA is listed in table 1.

\subsection{Possible formation process of PFA}

Based on our above experimental results, it can be concluded that PVP/FA ratio is an important factor in the formation of 

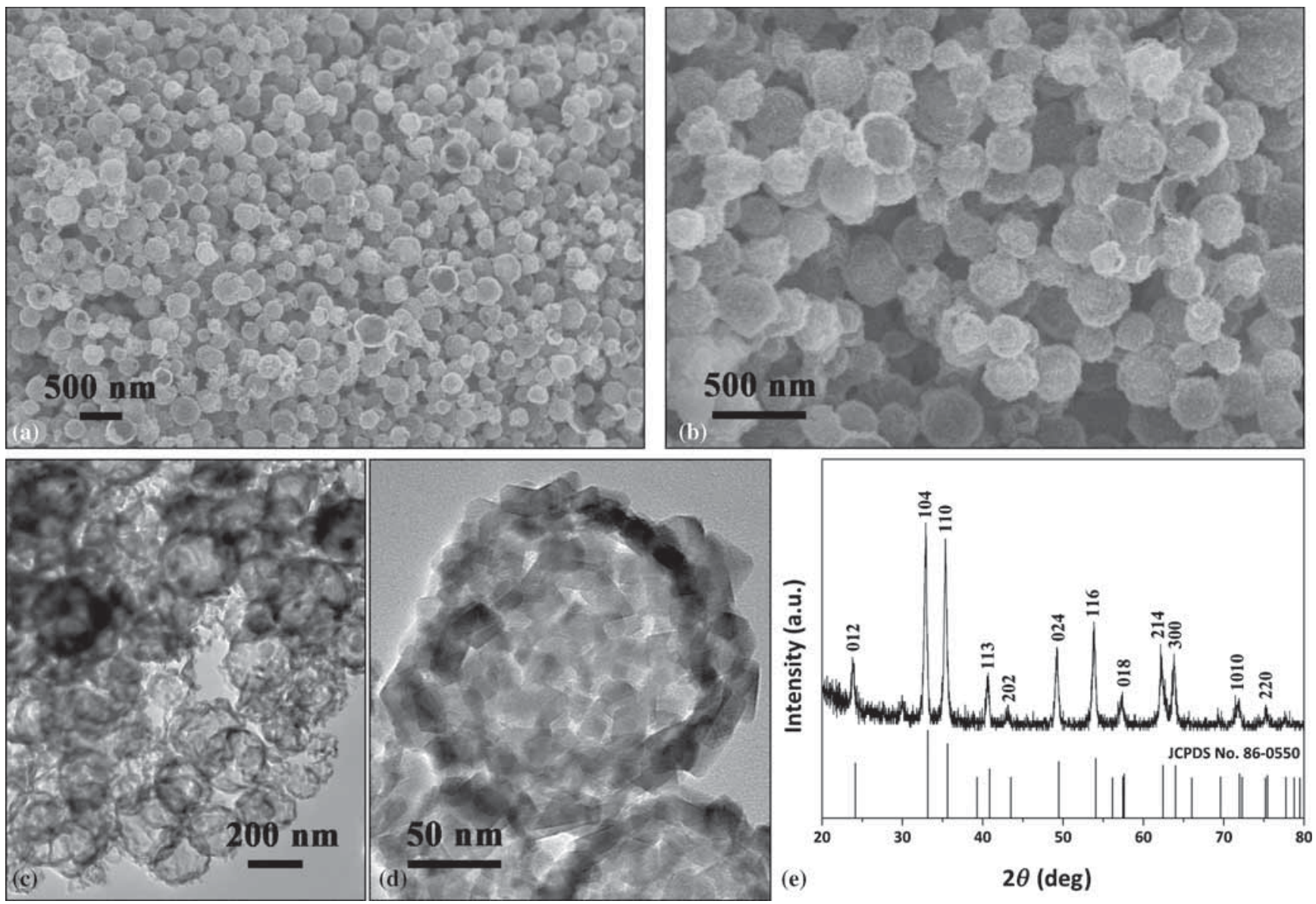

Figure 5. As-obtained $\alpha-\mathrm{Fe}_{2} \mathrm{O}_{3}$ hollow nanospheres: (a) general and (b) magnified SEM image; (c) TEM and (d) magnified TEM image; and (e) XRD pattern.

PFA nanospheres with uniform diameter and good dispersibility. In former studies, PVP merely served as a steric stabilizer to synthesize spherical polymer with good dispersibility. Alteration of PVP concentration did not affect the spherical morphology of polymer. ${ }^{32-34}$ In our system, different morphologies of PFA could be obtained by adjusting the PVP/FA ratio. Therefore, it was speculated that PVP can be used not only as a colloidal stabilizer but also as a control agent in the formation of PFA. As reported, pyrrole rings of PVP participate in an organic-organic interaction with the furan rings of FA due to $\pi-\pi$ stacking. ${ }^{35}$ As a result of this interaction, PVP may have a confinement effect on controlling the polymerization of FA, resulting in the formation of PFA with different morphologies.

In our reaction system, FA monomer polymerizes to form bulk PFA resin without PVP. Upon PVP addition, polymerization of FA is restricted by PVP owing to the interaction between the pyrrole rings and furan rings. When there is a higher amount of FA than PVP (for example, PVP/FA ratio of 0.4), PVP cannot completely confine polymerization of FA, resulting in the formation of large polyhedral PFA. However, when the amount of PVP is excessive, polymerization of FA is controlled completely by PVP. Such strong confinement is responsible for the good spherical morphology and dispersibility of products with uniform size. The abovementioned formation process of PFA can be illustrated as in figure 4.

Based on the above discussion, it can be concluded that spherical PFA can be obtained as long as polymerization of FA is entirely confined by excessive PVP. For example, although the amount of FA in experiment No. 9 was higher than No. 3 (table 1), products were mainly small PFA nanospheres instead of large polyhedrons thanks to the confinement effect of PVP. Therefore, the formation of PFA nanospheres based on the confinement effect of PVP should be reasonable.

\subsection{Characterization of $\alpha-\mathrm{Fe}_{2} \mathrm{O}_{3}$ hollow nanospheres}

PFA is often used as a precursor in preparing porous carbons or glassy carbons. ${ }^{6}$ Considering their functionalized surface and good dispersibility, our as-prepared PFA nanospheres are believed to be an ideal template for fabricating hollow nanostructures as well. ${ }^{36}$ The surface of as-prepared PFA nanospheres contains a large quantity of functional groups such as $-\mathrm{OH}$ and $-\mathrm{CH}_{2}-$ according to a previous FT-IR study. The functional groups on the surface of PFA are able to bind to metal cations through electrostatic 
interactions or coordination. In the subsequent calcinations process, the PFA core is removed, after which surface layers of metal cations are gradually densified to form oxide hollow nanospheres. The experimental result is in good agreement with our expectations. $\alpha-\mathrm{Fe}_{2} \mathrm{O}_{3}$ hollow nanospheres can be obtained successfully on a large scale.

The general SEM image (figure 5a) of the products shows exclusively spherical structures with diameters ranging from 200 to $250 \mathrm{~nm}$. The diameters of these nanospheres with rough surfaces are reduced to about $35 \%$ of the original diameters of the PFA nanospheres. As shown in the high-magnification SEM image (figure 5b), some broken spherical products can be observed, which shows the character of the hollow structure. This hollow structural character can be observed more clearly through TEM images of the products. TEM image (figure 5c) confirms that the hollowsphere structure of as-obtained products have a shell wall thickness of about 20-30 nm. A high-magnification TEM image (figure 5d) shows that the shell is formed from packed nanoparticles, which is consistent with the rough surface observed in the SEM images. Figure 5e displays the XRD pattern of the hollow nanospheres. All diffraction peaks can be readily indexed to hexagonal $\alpha-\mathrm{Fe}_{2} \mathrm{O}_{3}$ (JCPDS no. 860550), and sharpness of the peaks implies the high crystalline quality of the as-obtained sample.

As the functionalized surface of the as-prepared PFA nanospheres interacts with $\mathrm{Fe}^{3+}$ cations, an $\mathrm{Fe}(\mathrm{OH})_{3}$ layer will form on the surface of the PFA nanospheres when $\mathrm{NaOH}$ solution is added to the reaction system. The PFA core can be removed and the $\mathrm{Fe}(\mathrm{OH})_{3}$ layer decomposed during the calcination process, which will lead to the crystallization and condensation of $\alpha-\mathrm{Fe}_{2} \mathrm{O}_{3}$, resulting in the formation of hollow nanospheres. This can explain the shrinkage of the diameter of $\alpha-\mathrm{Fe}_{2} \mathrm{O}_{3}$ hollow nanospheres compared to that of PFA nanospheres. The formation process of $\alpha-\mathrm{Fe}_{2} \mathrm{O}_{3}$ hollow nanospheres could be extended to the preparation of many other metal oxide hollow nanospheres, with further work following on.

\section{Conclusions}

In summary, a facile hydrothermal approach has been developed for the fabrication of PFA nanospheres with good dispersibility. The experimental results demonstrated that the PVP/FA ratio imposes a significant influence on PFA morphology. According to the experimental discussions and former reports, a possible formation process of PFA nanospheres based on the confinement effect of PVP was proposed. Owing to interactions between the pyrrole rings and furan rings, PVP molecule acts as a control agent to restrict polymerization of FA. PFA nanospheres can be obtained only when polymerization of FA is entirely confined by PVP. Due to the functionalized surface, the as-prepared PFA nanospheres are an ideal template for the fabrication of hollow nanospheres, such as successful synthesis of $\alpha$ $\mathrm{Fe}_{2} \mathrm{O}_{3}$ hollow nanospheres. This feasible approach presents a new paradigm for the preparations of well-dispersed polymer nanospheres and hollow nanospheres.

\section{Acknowledgements}

This work was financially supported by the National Natural Science Foundation of China (No. 21201008 and No. 21501004) and the Anhui Provincial Natural Science Foundation (No. 1208085QB31).

\section{References}

1. Gandini A and Belgacem M N 1997 Prog. Polym. Sci. 221203

2. Gonzalez R, Martinez R and Ortiz P 1992 Makromol. Chem. 1931

3. Magalhaes W L E and da Silva R R 2004 J. Appl. Polym. Sci. 911763

4. Kherroub D E, Belbachir M and Lamouri S 2015 Bull. Mater. Sci. 3857

5. Sthel M, Rieumont J and Martinez R 1999 Polym. Test. 1847

6. Wang H T and Yao J F 2006 Ind. Eng. Chem. Res. 456393

7. Gandini A and Martinez R 1983 Makromol. Chem. 1841189

8. Pepper D C and Ryan B 1983 Makromol. Chem. 184395

9. Gonzalez R, Rieumont J, Figueroa J M, Siller J and Gonzalez H 2002 Eur. Polym. J. 38281

10. Ugelstad J, Stenstad P, Kilaas L, Prestvik W S, Rian A, Nustad K, Herje R and Berge A 1996 Macromol. Symp. 101491

11. Johnson S A, Ollivier P J and Mallouk T E 1999 Science 283 963

12. Pavel F M 2004 J. Dispersion Sci. Technol. 251

13. Zhang G Z, Niu A Z, Peng S F, Jiang M, Tu Y F, Li M and Wu C 2001 Acc. Chem. Res. 34249

14. Zhang Q, Remsen E E and Wooley K L $2000 \mathrm{~J}$. Am. Chem. Soc. 1223642

15. Butun V, Billingham N C and Armes S P 1998 J. Am. Chem. Soc. 12012135

16. Antonietti M, Bremser W and Pakula T 1995 Acta Polym. 4637

17. Mecerreyes D, Lee V, Hawker C J, Hedrick J L, Wursch A, Volksen W, Magbitang T, Huang E and Miller R D $2001 A d v$. Mater. 13204

18. Harth E, Van Horn B, Lee V Y, Germack D S, Gonzales C P, Miller R D and Hawker C J 2002 J. Am. Chem. Soc. 124 8653

19. Kumar K Y, Muralidhara H B, Nayaka Y A, Hanumanthappa H, Veena M S and Kumar S R K 2015 Bull. Mater. Sci. 38 271

20. Tan G X, Liao J W, Ning C Y and Zhang L 2012 J. Appl. Polym. Sci. 1253509

21. Munoz-Bonilla A, van Herk A M and Heuts J P A 2010 Polym. Chem. 1624

22. Lopez-Leon T, Ortega-Vinuesa J L, Bastos-Gonzalez D and Elaissari A 2006 J. Phys. Chem. B 1104629

23. Liu J, Wang H T, Cheng S A and Chan K Y 2004 Chem. Commun. 728

24. Gonzalez R, Figueroa J M and Gonzalez H 2002 Eur. Polym. J. 38287 
25. Wang Z, Lu Z, Huang X, Xue R and Chen L 1998 Carbon 36 51

26. Haas I, Shanmugam S and Gedanken A 2006 J. Phys. Chem. B 11016947

27. Choura M, Belgacem N M and Gandini A 1996 Macromolecules 293839

28. Barr J B and Wallon S B 1971 J. Appl. Polym. Sci. 151079

29. Fawcett A H and Dadamba W 1982 Makromol. Chem. 183 2799

30. Principe M, Ortiz P and Martinez R 1999 Polym. Int. 48 637
31. Chuang I S, Maciel G E and Myers G E 1984 Macromolecules 171087

32. Paine A J, Luymes W and Mcnulty J 1990 Macromolecules 23 3104

33. Lee J W, Ha J U, Choe S, Lee C S and Shim S E 2006 J. Colloid Interface Sci. 298663

34. Ye Q, He W D, Ge X W, Jia H T, Liu H R and Zhang Z C 2002 J. Appl. Polym. Sci. 862567

35. Yan Y, Yang H F, Zhang F Q, Tu B and Zhao D Y 2006 Small 2517

36. Sun X M, Liu J F and Li Y D 2006 Chem. Eur. J. 122039 